\title{
Decreased cervical cancer cell adhesion on nanotubular titanium for the treatment of cervical cancer
}

This article was published in the following Dove Press journal:

International Journal of Nanomedicine

5 March 2013

Number of times this article has been viewed

\author{
Jara Crear \\ Kim M Kummer \\ Thomas J Webster \\ School of Engineering, Brown \\ University, Providence, RI, USA
}

Correspondence: Thomas J Webster Chair and Professor, Department of Chemical Engineering, Northeastern University, Boston, MA 021I5 USA

Tel 6173736585

Fax 6173732209

Email th.webster@neu.edu

\begin{abstract}
Cervical cancer can be treated by surgical resection, chemotherapy, and/or radiation. Titanium biomaterials have been suggested as a tool to help in the local delivery of chemotherapeutic agents and/or radiation to cervical cancer sites. However, current titanium medical devices used for treating cervical cancer do not by themselves possess any anticancer properties; such devices act as carriers for pharmaceutical agents or radiation sources and may even allow for the growth of cancer cells. Based on studies, which have demonstrated decreased lung, breast, and bone cancer cell functions on nanostructured compared to nanosmooth polymers, the objective of the present in vitro study was to modify titanium to possess nanotubular surface features and determine cervical cancer cell adhesion after 4 hours. Here, titanium was anodized to possess nanotubular surface features. Results demonstrated the ability to decrease cervical cancer cell adhesion by about a half on nanotubular compared to currently used nanosmooth titanium (without the use of chemotherapeutics or radiation), opening up numerous possibilities for the use of nanotubular titanium in local drug delivery or radiation treatment of cervical cancer.
\end{abstract}

Keywords: cervical cancer, titanium, nanotubular, cell density

\section{Introduction}

Cervical cancer is a malignant neoplasm arising from cells originating in the cervix uteri. ${ }^{1}$ Treatment usually consists of surgery in early stages and chemotherapy and/or radiotherapy in more advanced stages. ${ }^{1}$ The treatment of cervical cancer varies worldwide largely due to variances in disease burden in developed and developing nations, access to surgeons skilled in radical pelvic surgery, and the need to not disrupt female reproductive organs in certain nations or cultures. Because cervical cancers are radiosensitive, radiation may be used in all stages where surgical options do not exist. ${ }^{1}$

Specifically, microinvasive cervical cancer (stage IA) may be treated by hysterectomy or removal of the whole uterus including part of the vagina. ${ }^{1}$ For stage IA2, the lymph nodes are removed as well. ${ }^{1}$ For $1 \mathrm{~A} 1$ disease, a cone biopsy is considered curative. Early stages (IB1 and IIA less than $4 \mathrm{~cm}$ ) can be treated with radical hysterectomy with removal of the lymph nodes or radiation therapy. ${ }^{1}$ Radiation therapy is either given as external beam radiotherapy to the pelvis or internally (called brachytherapy). ${ }^{1}$ Patients treated with surgery who have high-risk features found on pathological examinations are also given radiation therapy with or without chemotherapy in order to reduce the risk of relapse. Larger early stage tumors (IB2 and IIA more than $4 \mathrm{~cm}$ ) may also be treated with radiation therapy and cisplatin-based chemotherapy, hysterectomy (which then usually requires adjuvant radiation therapy), or cisplatin chemotherapy followed by hysterectomy. ${ }^{1}$ Lastly, 
advanced stage tumors (IIB-IVA) are treated with radiation therapy and cisplatin-based chemotherapy. ${ }^{1}$

To assist in cervical cancer treatment, biomaterials have been used as a carrier of pharmaceutical agents as well as materials that emit ionizing radiation energy locally. ${ }^{2}$ Thus, such biomaterials are often inserted into appropriate anatomical locations to release drugs or radiation. Healthy cells will also be affected by radiation, but they are usually robust enough to repair themselves and recover normal life cycles. Due to its biocompatibility and widespread use in the medical device industry, titanium has often been used and proposed for cervical cancer treatment. ${ }^{2-4}$ Specifically, titanium is currently clinically used to reduce magnetic resonance imaging artifacts and distortion for the diagnosis of cervical cancer and is currently being proposed as a carrier for drugs and radiation for the treatment of cervical cancer. However, titanium possesses no ability to decrease cancer cell functions unless radiation or drugs are released and, in fact, titanium may even promote cervical cancer cell growth on its surface.

Due to previous studies that have demonstrated decreased bone, lung, and breast cancer cell functions on polymers with select nanoscale surface features, ${ }^{5-7}$ the objective of this study was to create nanoscale surface features on titanium and determine for the first time, cervical cancer cell responses. Results demonstrated a quick and easy way to modify titanium to possess nanoscale surface features that can decrease cervical cancer cell adhesion and may be a better drug or radiation carrier than unmodified titanium.

\section{Materials and methods}

\section{Material preparation}

Nanotubular titanium was synthesized using $99.5 \%$ pure titanium foils ( $0.25 \mathrm{~mm}$ thick; AlfaAesar, Ward Hill, MA, USA) cut into $0.8 \times 0.8 \mathrm{~mm}$ squares. Titanium samples were connected to an electrochemical cell with a two-electrode configuration. A platinum mesh (AlfaAesar) was used as the cathode and a titanium specimen was used as the anode. These platinum and titanium samples were connected to a DC power supply (3645A DC power supply; Circuit Specialists Inc, Mesa, AZ, USA) through copper wires (AlfaAesar).

In order to create nanotubular titanium with 20,40, 60, and $80 \mathrm{~nm}$ tube diameters, the applied anodization voltages were changed to $5,10,15$, and $20 \mathrm{~V}$, respectively, in a $0.5 \%$ hydrofluoric acid (HF) electrolyte solution for an anodization time of 30, 30, 20, and 15 minutes, respectively. Once this was completed, the anodized samples were sterilized by rinsing with acetone, $70 \%$ ethanol, and $\mathrm{ddH}_{2} \mathrm{O}$ for 10 minutes each. Once samples were sterilized, they were then hydrothermally treated (heat treated) in a furnace (Lindberg/Blue furnace; VWR, Radnor, PA, USA) at $500^{\circ} \mathrm{C}$ for 2 hours with a heating/cooling rate of $3^{\circ} \mathrm{C} /$ minute at ambient pressure since previous studies have demonstrated the elimination of fluorine through such processes. ${ }^{8}$ Lastly, prior to cell experiments, anodized samples and conventional titanium samples (untreated) were sterilized using an autoclave process.

\section{Material characterization}

\section{Scanning electron microscopy (SEM)}

Surface characterization of the specimens was conducted using a LEO 1530 VP FE-4800 field-emission scanning electron microscope (Zeiss, Peabody, MA, USA). A $5 \mathrm{kV}$ accelerating voltage was chosen for SEM analysis and the micrographs were captured using secondary electrons collected with an in-lens detector. No sputtering was used to image the surfaces of the conventional or anodized titanium substrates.

\section{Atomic force microscopy (AFM)}

For surface roughness measurements, an Asylum Research AFM (Santa Barbara, CA, USA) was used to scan the conventional and anodized titanium substrates. Each sample was analyzed in ambient air under noncontact mode using a silicone ultrasharp cantilever (probe tip radius of $10 \mathrm{~nm}$; MikroMasch, Wilsonville, OR, USA). $1 \times 1 \mu \mathrm{m}$ and $90 \times 90 \mu \mathrm{m}$ AFM fields were analyzed and the scan rate was chosen as $1 \mathrm{~Hz}$. Image analysis software (IgorPro, Seattle, WA, USA) was used to generate micrographs and to quantitatively compare the root-mean-square roughness (RMS) of the conventional and the anodized titanium substrates. RMS is the estimated standard deviation of the bidimensional Gaussian-type distribution of heights around the mean value of the collected points.

\section{Chemical and crystallinity analysis (ESCA and XRD)}

For chemical analysis of the top surface layer of the conventional and anodized titanium samples, electron spectroscopy for chemical analysis (ESCA) was performed using a Perkin Elmer 5500 Multitechnique Surface Analyzer System (Waltham, MA, USA). An aluminum K-alpha monochromatized X-ray source was used to stimulate photoemission of the inner shell electrons on the surfaces. The energy of these electrons was then recorded and analyzed for all substrates. Wide scans of both conventional and anodized titanium were used to generate low-resolution spectra to identify and quantify the percentage of different elements up to a depth of $100 \AA$. X-ray diffraction (XRD) was also conducted in this study according to standard procedures. There was no sputter cleaning done to any of the samples before these characterization methods. 


\section{Contact angle analysis}

Water contact angles were investigated using a drop shape analysis system (EasyDrop; Kruss, Hamburg, Germany). The contact angle of $3 \mu \mathrm{L}$ sessile droplets was measured at two locations on each conventional titanium and anodized nanotubular titanium substrate.

\section{Cell studies}

Human cervical cancer cells (CCL-2 HeLa cells, population numbers 3 and 4; American Type Culture Collection, Manassas, VA, USA) were cultured in Dulbecco's modified Eagle's medium (DMEM), high glucose (HyClone; Gibco, Grand Island, NY, USA), supplemented with $10 \%$ fetal bovine serum (FBS) and $1 \%$ penicillin/streptomycin in a $37^{\circ} \mathrm{C}$, humidified, $5 \% \mathrm{CO}_{2}$ environment. The effect of the nanotubular titanium surface features on HeLa cell adhesion was determined using the MTT (3-(4,5-dimethyl-2-thiazolyl)2,5-diphenyl-2H-tetrazolium bromide) assay (Invitrogen, Carlsbad, CA, USA). Samples were sterilized by autoclave sterilization. Prior to cell seeding, all samples were rinsed with phosphate-buffered solution (PBS) for five minutes. Cells were seeded at 3500 cells $/ \mathrm{cm}^{2}$ per substrate in a 24-well plate and were allowed to adhere for 4 hours in a $37^{\circ} \mathrm{C}$, humidified, $5 \%$
$\mathrm{CO}_{2}$ environment. After 4 hours, the substrates were rinsed in PBS to remove nonadherent cells and were transferred to fresh media in a clean 24 -well plate. Next, $150 \mu \mathrm{L}$ of the MTT solution was added to each well and incubated for 4 hours. Viable cells reduced the yellowish tetrazolium into insoluble purple formazan crystals that was then dissolved with $1 \mathrm{~mL}$ of an isopropanol/ $\mathrm{HCl}$ solution. After 12 hours of incubation, the solutions were transferred into a clean 96-well plate and absorbance at $570 \mathrm{~nm}$ was read using a microplate reader (SoftMax Pro, Minneapolis, MN, USA) to obtain optical densities. A standard curve was created to determine cell numbers from MTT adsorbance values.

\section{Statistical analysis}

All experiments were conducted in triplicate and repeated three different times. Differences between means were determined using analyses of variance followed by Student's $t$-tests.

\section{Results and discussion \\ Material characterization}

\section{Micro- and nanostructure characterization}

Scanning electron microscopy (SEM) images of conventional titanium and anodized nanotubular titanium are shown below

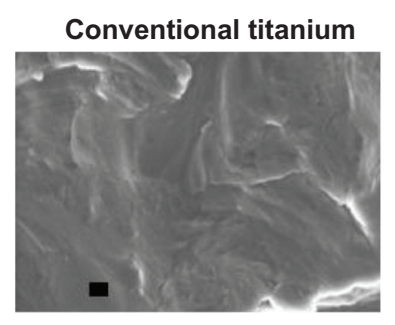

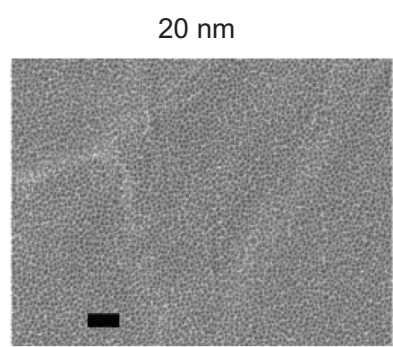

$60 \mathrm{~nm}$

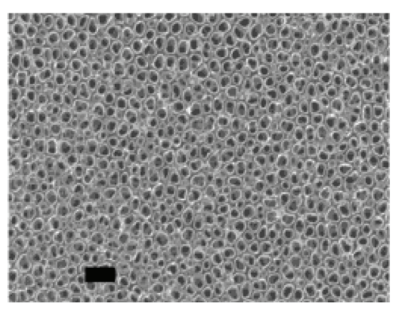

$40 \mathrm{~nm}$

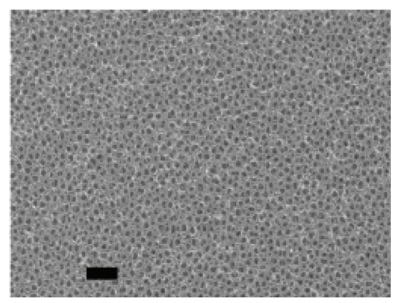

$80 \mathrm{~nm}$

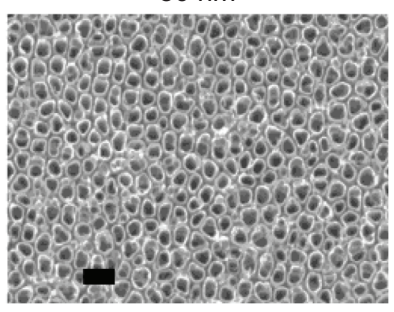

$200 \mathrm{~nm}$

Figure I SEM images of conventional titanium and anodized titanium possessing 20, 40, 60 and $80 \mathrm{~nm}$ diameter tubes. Note: Scale bars $=100 \mathrm{~nm}$. 
in Figure 1. As expected, SEM characterization revealed that the diameter of the titanium nanotubular features varied between 20 and $80 \mathrm{~nm}$ diameter tubes. AFM images revealed additional distinct topographical differences between some of the substrates of interest in this study (Figure 2). As expected due to this change in surface features, RMS roughness was larger on the anodized nanotubular titanium surfaces. AFM scans also showed higher RMS values for the titanium anodized to possess $20 \mathrm{~nm}$ diameter nanotubes compared to both the titanium anodized to possess $80 \mathrm{~nm}$ diameter nanotubes and conventional titanium (Figure 2). Table 1 summarizes the RMS values.

\section{Surface chemistry analysis (ESCA)}

In Table 2, the chemical compositions of the conventional and the anodized nanotubular titanium samples used in this study are compared. One significant difference between the samples was the carbon content on the surface of anodized
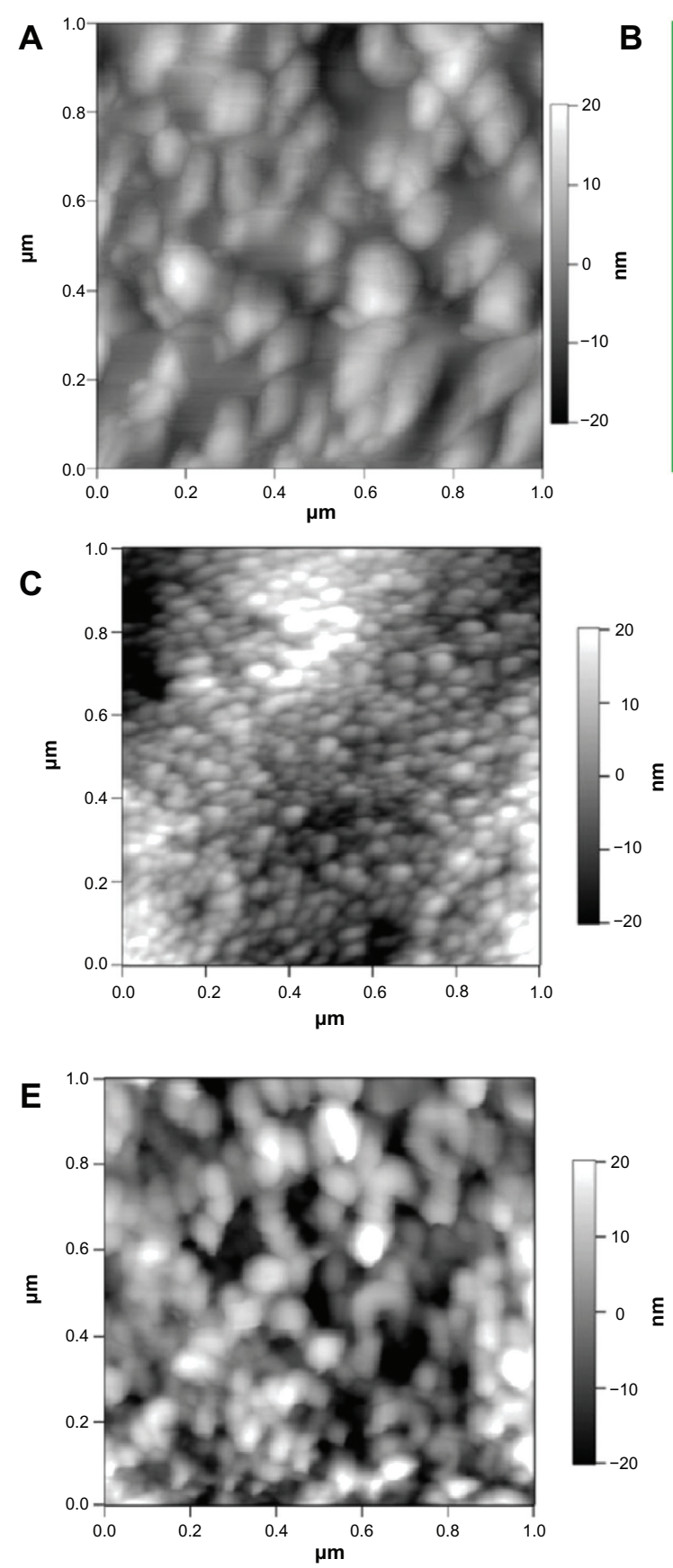
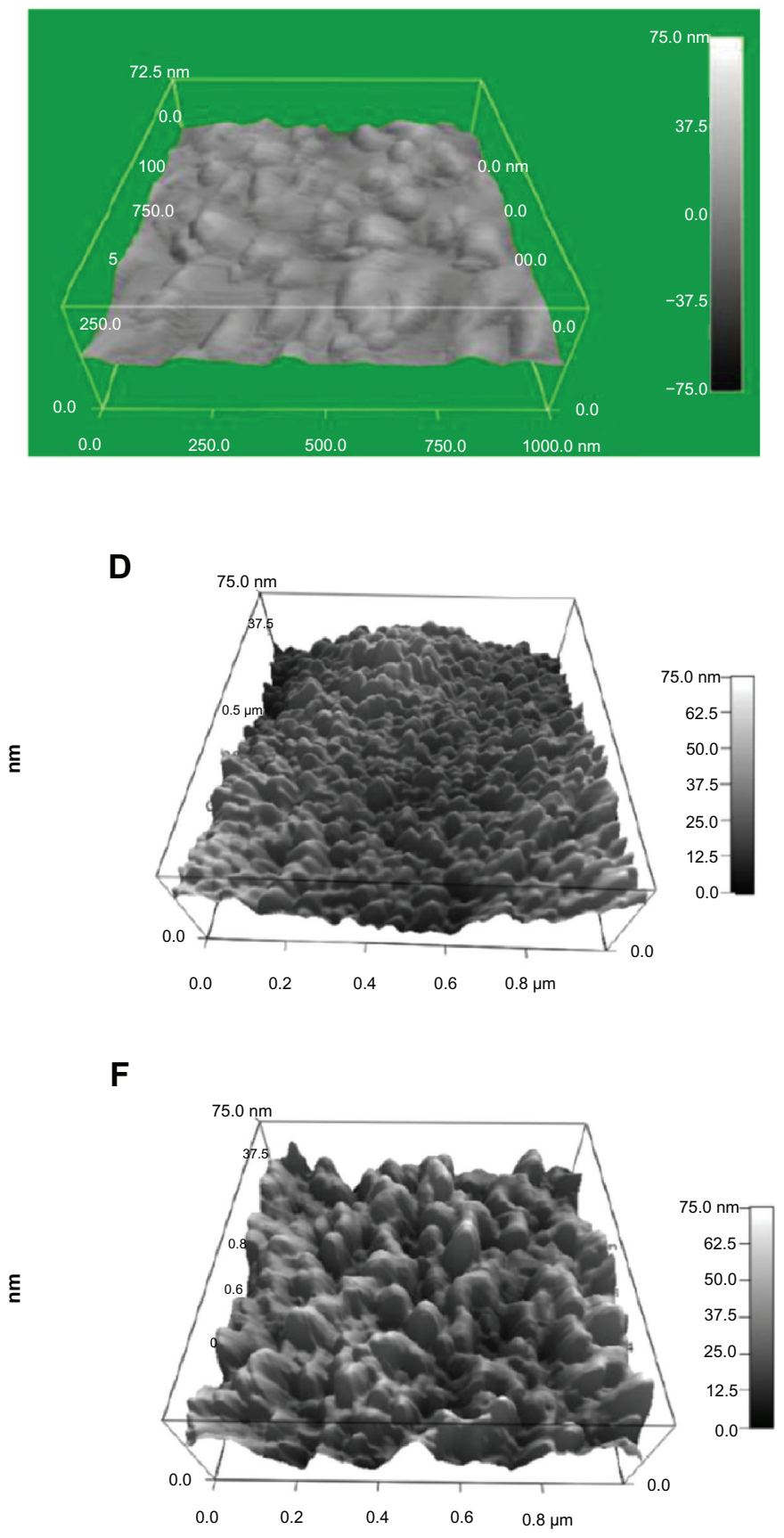

Figure 2 Atomic force micrographs showing the nanotopography of (A and $\mathbf{B})$ conventional, $(\mathbf{C}$ and $\mathbf{D}) 20 \mathrm{~nm}$, and (E and $\mathbf{F}) 80 \mathrm{~nm}$ titanium. 
Table I Quantitative data obtained from atomic force microcsopy analysis indicating that substrates created by anodization contained more nanofeatured surfaces compared to conventional titanium

\begin{tabular}{ll}
\hline Titanium substrate & Root-mean-squares value $\mathbf{( n m})$ \\
\hline Conventional & $6.57 \pm 0.37$ \\
$20 \mathrm{~nm}$ & $9.97 \pm 0.83$ \\
$40 \mathrm{~nm}$ & $9.54 \pm 0.66$ \\
$60 \mathrm{~nm}$ & $9.37 \pm 0.43$ \\
$80 \mathrm{~nm}$ & $9.01 \pm 0.40$ \\
\hline
\end{tabular}

nanotubular titanium and conventional titanium. This carbon layer is derived from the hydrocarbons deposited onto the titanium substrates, which was not eliminated during the vigorous cleaning process. Along with carbon, oxygen concentration on the varying nanotubular titanium surfaces showed some important patterns. All of the nanotubular anodized titanium samples had higher concentrations of oxygen compared to conventional samples. From the ESCA results, it can be concluded that the surface chemistry of the samples showed key differences that may have played a role in cellular responses to be described below. In addition, these results reveal that the trends of altered surface chemistry as the titanium nanotubular dimensions change need to be further investigated in future studies.

\section{Crystal structure (XRD analysis)}

XRD results are shown in Figure 3. The titanium anodized to possess $80 \mathrm{~nm}$ diameter nanotubes had an anatase peak but did not show any rutile peaks, yet, as the diameter of the tubes decreased to $20 \mathrm{~nm}$, the formation of rutile became more significant. In fact, the titanium anodized to possess $20 \mathrm{~nm}$ nanotubes did not show any indication of an anatase phase but showed indications of the rutile crystalline phase. The titanium anodized to possess 40 and $60 \mathrm{~nm}$ nanotube diameters were similar to the $80 \mathrm{~nm}$ diameter titanium samples (data not shown).

Other researchers observed similar results for titanium oxide layers. ${ }^{9} 10$ For example, Gribb et al, observed an increase in the kinetics of anatase to rutile phase transformation when
Table 2 Atomic percentages of elements given by electron spectroscopy for chemical analysis of anodized nanotubular titanium samples of interest to the current study

\begin{tabular}{llll}
\hline Titanium substrate & $\mathbf{T i}$ & $\mathbf{O}$ & $\mathbf{C}$ \\
\hline Conventional & 14.93 & 43.99 & 41.07 \\
$20 \mathrm{~nm}$ & 22.08 & 68.19 & 9.73 \\
$40 \mathrm{~nm}$ & 21.89 & 65.88 & 12.23 \\
$60 \mathrm{~nm}$ & 23.33 & 62.30 & 14.37 \\
$80 \mathrm{~nm}$ & 24.57 & 54.47 & 20.96 \\
\hline
\end{tabular}

the anatase crystals were finely crystalline, ie, coarsely grained anatase remained $100 \%$ anatase upon heat treatment at $700^{\circ} \mathrm{C}$ for 24 hours, yet, nanocrystalline anatase transformed to $80 \%$ rutile after 24 hours at $525^{\circ} \mathrm{C} .{ }^{10}$ Gribb et al explained the enhancement in phase transformation from anatase to rutile as an increase in favorable nucleation sites at smaller crystallite sizes. Perhaps the same phenomenon occurred for anodized titanium here and this feature size difference affected phase transformation, where the transformation kinetics were much faster than that for the $20 \mathrm{~nm}$ compared to the 40,60 , and $80 \mathrm{~nm}$ diameter nanotubular titanium. Other possible reasons for these crystalline phase transformations include: (i) the presence of different titanium oxide compounds on the surfaces, which could potentially give different crystal structures at high temperatures and (ii) the presence of a [TiF6] $]^{2-}$ compound due to titanium anodization in HF; high temperature decomposition was not investigated here, but should be the focus of future studies.

Furthermore, the phase transformation kinetics could have transformed the oxide layer into different crystalline structures at the end of heating or perhaps resulted in faster oxygen transport during heat treatment. The $80 \mathrm{~nm}$ diameter nanotubular structures compared to the $20 \mathrm{~nm}$ diameter nanotubular structures might have decomposed $\left[\mathrm{TiF}_{6}\right]^{2-}$ to different oxide chemistries, which could further transform into anatase or rutile; all of these events could be influential factors that affected the formation of the different crystal phases observed in this study and need to be further studied.
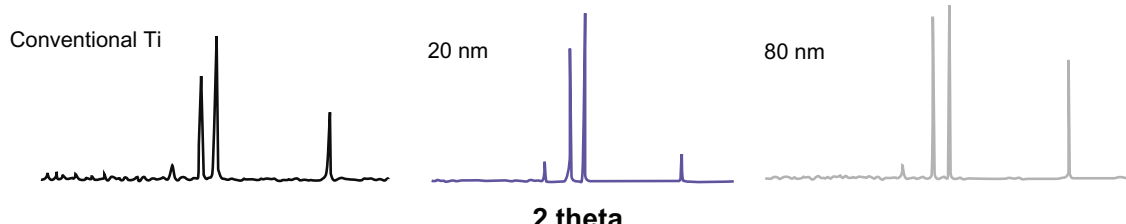

2 theta

Figure 3 X-ray diffraction spectra of conventional titanium, $20 \mathrm{~nm}$, and $80 \mathrm{~nm}$ samples. Note: Forty and $60 \mathrm{~nm}$ samples were similar to $80 \mathrm{~nm}$ samples. 


\section{Surface hydrophilicity analysis}

Water contact angles were used to assess the hydrophilicity of the samples of interest to this study (Figure 4). The most hydrophobic sample was conventional titanium with a water contact angle that was the highest while all the anodized nanotubular titanium samples showed contact angles below $30^{\circ}$. The most hydrophilic sample was titanium anodized to possess $20 \mathrm{~nm}$ diameter nanotubes. Overall, these contact angles correlate well to the ESCA results shown above. Specifically, as carbon content increased and oxygen content decreased on the conventional and anodized nanotubular titanium surfaces, there was an increase in water contact angle.

\section{Cervical cancer cell responses}

Lastly, the lowest cervical cancer adhesion was observed on the titanium with $60 \mathrm{~nm}$ diameter tubes, which was half of that of all the other substrates tested in the present study (Figure 5). Since the adhesion of cancer cells is a necessary prerequisite for their growth and migration, such results show great promise for the $60 \mathrm{~nm}$ anodized nanotubular titanium samples to decrease cervical cancer cell responses compared to the nanosmooth titanium currently used to locally deliver pharmaceutical agents or radiation and, thus, should be further considered for a wide range of anticancer treatment.

It is intriguing to wonder why titanium anodized to possess $60 \mathrm{~nm}$ tubes decreased cervical cancer cell adhesion more than any other sample studied here. As mentioned, previous studies have demonstrated promise for decreasing cancer cell responses on polymers with select nanoscale surface features, specifically $23 \mathrm{~nm}$ diameter hemispherical structures compared to those larger than $200 \mathrm{~nm} .^{5-7}$ Nanopatterned poly(lactic-co-glycolic acid) (PLGA) surfaces with similar surface chemistry but different topographies decreased breast and lung cancer cell density and vascular endothelial

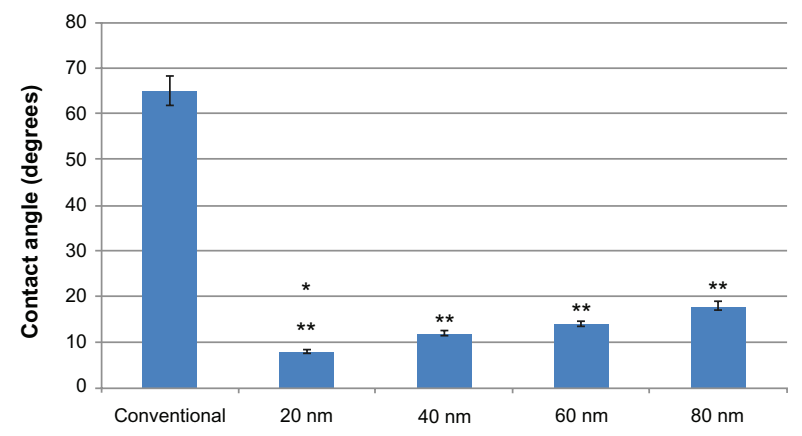

Figure 4 Water contact angles of the samples used in this research. Notes: Values are mean \pm standard error of mean, $\mathrm{N}=3$. $* P<0.01$ compared to all other samples; **P $<0.01$ compared to controls.

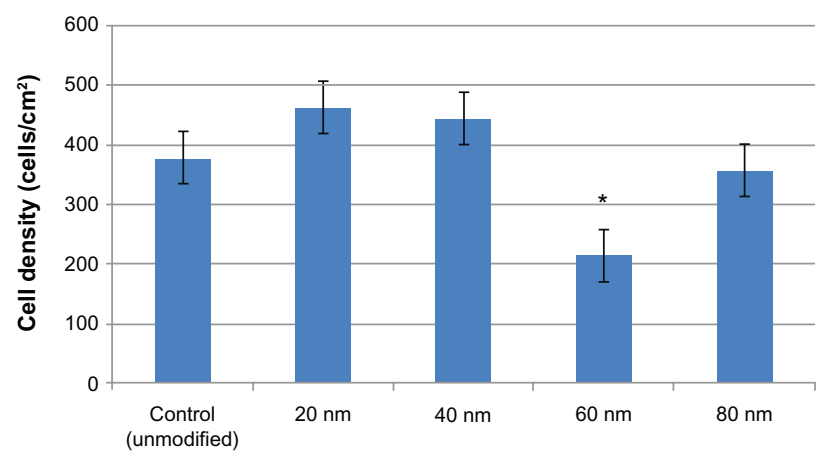

Figure 5 Decreased cervical cancer cell adhesion on the $60 \mathrm{~nm}$ nanotubular titanium.

Notes: Data $=$ mean \pm standard error of the mean; $\mathrm{N}=3 ; * P<0.01$ (compared to all others).

cell factor (VEGF, a growth factor which promotes tumor angiogenesis) synthesis compared to nanosmooth or flat PLGA samples. This was in contrast to healthy breast and lung cell density which was greater on PLGA with $23 \mathrm{~nm}$ surface features than on flat PLGA. Increased healthy bone cell functions but decreased osteosarcoma cell functions have also been measured. In this manner, those studies coupled with the present study open the possibility of using such polymer nanotopographies for a wide range of anticancer applications including local drug and radiation delivery.

While it is not known at this time how nanoscale surface features decrease cancer cell responses, the $60 \mathrm{~nm}$ tubular titanium surface fabricated here may have possessed the ideal surface wettability to attract the initial adsorption of proteins from serum that decrease cancer cell attachment. This mechanism has been detailed for the ability of nanotubular titanium to increase bone growth as studies have measured significantly greater adsorption of vitronectin and fibronectin on nanotubular surfaces compared to flat titanium. ${ }^{11}$ Other surface properties, such as crystallinity measured by XRD and chemistry measured by ESCA, may have also influenced surface energetics to promote the adsorption of proteins know to inhibit cervical cancer cell adhesion. Although future studies will determine the exact mechanism by which such select nanosurface features decrease cervical cancer cell functions, this study adds to the number of studies which have demonstrated significant promise for the use of anodized nanotubular titanium in orthopedics, amputee devices, vascular stents, bladder stents, and antibacterial applications. ${ }^{11-17}$ Importantly, in all of those studies, increased functions of vascular cells, bladder cells, bone cells, etc have been measured demonstrating that these $60 \mathrm{~nm}$ tubular titanium structures may only be harmful to cervical cancer cells, yet healthy to other noncancerous cells. 


\section{Conclusion}

Titanium biomaterials have been suggested as a tool to help in the local delivery of chemotherapeutic agents and/or radiation to cervical cancer sites. However, current titanium medical devices used for treating cervical cancer do not by themselves possess any anticancer properties and may even allow for the growth of cancer cells. Results of this study demonstrated the ability to decrease cervical cancer cell adhesion after 4 hours by about a half on anodized nanotubular titanium compared to currently used nanosmooth titanium (without the use of chemotherapeutics or radiation) opening up numerous possibilities for the use of nanotubular titanium in local drug or radiation treatment of cervical cancer.

\section{Acknowledgment}

The authors would like to thank the Hermann Foundation for funding.

\section{Disclosure}

The authors report no conflicts of interest in this work.

\section{References}

1. Kumar V, Abbas AK, Fausto N, Mitchell RN. Robbins Basic Pathology, 8th ed. Philadelphia, PA: Saunders Elsevier; 2007:718-721.

2. Kim Y, Muruganandham M, Modrick JM, Bayouth JE. Evaluation of artifacts and distortions of titanium applicators on 3.0-Tesla MRI: feasibility of titanium applicators in MRI-guided brachytherapy for gynecological cancer. Int J Radiat Oncol Biol Phys. 2011;80(3):947-955.

3. Leonard KL, DiPetrillo TA, Munro JJ, Wazer DE. A novel ytterbium-169 brachytherapy source and delivery system for use in conjunction with minimally invasive wedge resection of early-stage lung cancer. Brachytherapy. 2011;10(2):163-169.
4. Andersen CE, Nielsen SK, Lindegaard JC, Tanderup K. Time-resolved in vivo luminescence dosimetry for online error detection in pulsed dose-rate brachytherapy. Med Phys. 2009;36(11):5033-5043.

5. Zhang L, Chun Y, Webster TJ. Decreased lung carcinoma cell density on select polymer nanometer surface features for lung replacement therapies. Int J Nanomedicine. 2010;5:269-275.

6. Wang Y, Webster TJ. Increased healthy osteoblasts to osteosarcoma cell ratios on specific PLGA nanopatterns. Int J Nanomedicine. 2012; In press.

7. Zhang L, Chun Y, Webster TJ. Decreased breast carcinoma cell density on select polymer nanometer surface features. Nanotechnology. 2012; In press.

8. Ercan B, Kummer K, Tarquinio K, Webster TJ. Decreased Staphylococcus aureus biofilm growth on anodized nanotubular titanium and the effect of electrical stimulation. Acta Biomater. 2011;7(7):3003-3012.

9. Macak JM, Tsuchiya H, Ghicov A, et al. TiO(2) nanotubes: Selforganized electrochemical formation, properties and applications. Curr Opin Solid State Mat Sci. 2007;11:3-18.

10. Gribb AA, Banfield JF. Particle size effects on transformation kinetics and phase stability in nanocrystalline $\mathrm{TiO}_{2}$. Am Miner. 1997;82: 717-728.

11. Yao C, Slamovich EB, Webster TJ. Enhanced osteoblast functions on anodized titanium with nanotube-like structures. J Biomed Mater Res Part A. 2008;85A:157-166.

12. Puckett $\mathrm{S}$, Pareta R, Webster TJ. Nano rough micron patterned titanium for directing osteoblast morphology and adhesion. Int J Nanomedicine. 2008;3:229-241.

13. Ercan B, Kummer KM, Tarquinio KM, Webster TJ. Decreased Staphylococcus aureus biofilm growth on anodized nanotubular titanium and the effect of electrical stimulation. Acta Biomater. 2011;7:3003-3012.

14. Ercan B, Taylor E, Alpaslan E, Webster TJ. Diameter of titanium nanotubes influences anti-bacterial efficacy. Nanotechnology. 2011:22:295102.

15. Puckett SD, Taylor E, Raimondo T, Webster TJ. The relationship between the nanostructure of titanium surfaces and bacterial attachment. Biomaterials. 2010;31:706-713.

16. Alpaslan E, Ercan B, Webster TJ. Anodized $20 \mathrm{~nm}$ diameter nanotubular titanium for improved bladder stent applications. Int J Nanomedicine. 2011;6:219-225.

17. Puckett SD, Lee PP, Ciombor DM, Aaron RK, Webster TJ. Nanotextured titanium surfaces for enhancing skin growth on transcutaneous osseointegrated devices. Acta Biomater. 2010;6:2352-2362.
International Journal of Nanomedicine

\section{Publish your work in this journal}

The International Journal of Nanomedicine is an international, peerreviewed journal focusing on the application of nanotechnology in diagnostics, therapeutics, and drug delivery systems throughout the biomedical field. This journal is indexed on PubMed Central, MedLine, CAS, SciSearch ${ }^{\circledR}$, Current Contents ${ }^{\circledR} /$ Clinical Medicine,

\section{Dovepress}

Journal Citation Reports/Science Edition, EMBase, Scopus and the Elsevier Bibliographic databases. The manuscript management system is completely online and includes a very quick and fair peer-review system, which is all easy to use. Visit http://www.dovepress.com/ testimonials.php to read real quotes from published authors. 